\title{
Shoe microclimate: An objective characterisation and subjective evaluation
}

\begin{abstract}
Shoe microclimate (temperature and humidity) has been suggested to contribute to perceptions of foot thermal comfort. However, limited data is available for perceptual responses in relation to shoe microclimate development both over time and within different areas of the shoe. This study evaluates perceptions of foot thermal comfort for two running shoes different in terms of air permeability in relation to temporal and spatial characteristics of shoe microclimate. The temporal characteristics of shoe microclimate development were similar for both shoes assessed. However, higher temperatures and humidity were observed for the less permeable shoe. Changes to shoe microclimate over time and differences between shoes were perceivable by the users. This study provides the most detailed assessment of shoe microclimate in relation to foot thermal comfort to date, providing relevant information for footwear design and evaluation.
\end{abstract}

Key Words: Footwear, Microclimate: Thermal Comfort 


\section{Introduction}

There has always been a demand for footwear which is both functional and comfortable. Yet the perception of comfort is complex and multi-factorial based on the interaction of factors which affect foot function during activity (Goonetilleke and Luximon 2001). These interactions can be linked to mechanical and thermal properties of shoes.

Thermal properties of shoes are typically attributed to the level of insulation and vapour resistance provided and the development of the shoe microclimate. Like clothing, shoes represent a barrier for heat and vapour transfer between the skin and the external environment. Thus when the wearer's dry and evaporative heat loss pathways are limited, heat and moisture may build within the air layers between the skin and the footwear (Havenith et al. 1990; Bouskill et al. 2002). Consequently, heat loss from the skin to the shoe is reduced as the gradient becomes smaller ( $\mathrm{Lu}$ et al. 2013). The development of the shoe microclimate has therefore been suggested to have a decisive influence on the users sensations and perceptions of thermal comfort (Arezes et al. 2013; Irzmanska et al. 2013; Shimazaki and Murata 2015). The materials used within shoes and socks, the air they enclose and the air bound on the outside of the material layers are therefore important (Havenith 2002).

Shoe microclimate development has been investigated in protective and hiking footwear but has been restricted to mechanical testing using thermal foot manikins (Schols et al. 2004) or assessment of temperature and relative humidity at one or two locations within footwear during physical activity. Under constant moderate climate conditions $\left(23-25^{\circ} \mathrm{C}, 50 \% \mathrm{RH}\right)$ and with exercise, in-shoe temperature and in-shoe relative humidity are reported to range between $27-37^{\circ} \mathrm{C}$ and $67-96 \%$ respectively (Bertaux et al. 2010; Irzmanska et al. 2013; Irzmańska 2015). Based on these data, foot skin temperatures (foot $T_{s k}$ ) have been reported to exceed $34^{\circ} \mathrm{C}$ resulting in perceptions of thermal discomfort. The combination of physical exercise and high temperatures inside footwear has also been shown to add strain to the cardiovascular system in the lower extremities, increasing blood flow by 33\% from rest (Irzmanska et al. 2013). Further consequences of high temperatures and levels of moisture inside shoes include tinea pedis, commonly known as athlete's foot. Primarily associated as a common problem for individuals who use public facilities such as saunas and swimming pools, tinea pedis also represents a problem for those who participate in 
physical activity where the feet are enclosed in shoes for extended periods of times i.e. marathon runners (Auger et al. 1993). Intermediate levels of moisture have also been shown to increase coefficients of friction which have been found to influence the probability of blister formation (Sulzberger et al. 1966). Changes to the shoe microclimate therefore encourage the growth of microorganisms which can lead to odour development and to poor foot health.

Knowledge regarding the subjective evaluation of shoe microclimate is limited, with little published information available. Although subjective perception of foot $T_{s k}$ may not always coincide with measured foot $T_{s k}$ (Barkley et al. 2011), local discomfort has been attributed to elevations in temperature rather than elevations in the moisture content both within hiking boots (Arezes et al. 2013) and within sock and boot liner materials worn within protective footwear (Irzmanska et al. 2013). The influence moisture has on foot comfort therefore requires further investigation as it is unknown whether changes in temperature and/or humidity help an individual in determining perceptions of thermal comfort.

Despite the impact shoe microclimate has on foot health and foot thermal comfort, to our knowledge no quantitative shoe microclimate data is available over time, within different areas of the shoe or in relation to perceptual responses specifically for sports related footwear. With exercise, metabolic heat generation and sweat rates are high and so balancing the amount of heat supplied to or generated by the feet with heat loss becomes crucial. Currently, only changes to foot $T_{s k}$ during running have been reported (Barkley et al. 2011; Shimazaki and Murata 2015; Shimazaki et al. 2015). During a 30 minute running bout at $12 \mathrm{~km} \mathrm{hr}^{-1}$ temperature elevations from rest of $8.2^{\circ} \mathrm{C}$ were observed at the heel and $4.8^{\circ} \mathrm{C}$ at the neck of the big toe, foot regions associated with high contact loads and pressure during running (Shimazaki and Murata 2015). Increased ventilation within running shoes has also been shown to produce a cooling effect, reducing foot $T_{s k}$ elevations, particularly on the arch of the foot (Shimazaki et al. 2015). How the development of shoe microclimate influences perceptual responses however has not been investigated. As commercial communities seek to improve footwear comfort, assessments of shoe microclimate in running footwear would therefore be valuable. 
The aim of this study was threefold: (i) to objectively investigate temporal and spatial characteristics of shoe microclimate in running footwear, (ii) to determine whether shoe microclimate is affected by the level of shoe permeability and (iii) to determine whether changes to shoe microclimate are perceivable. The evaluation of footwear thermal properties based upon objective and subjective parameters will provide valuable information for footwear evaluation and design. 


\section{Method}

\subsection{Participants}

Ten healthy females [age: $24 \pm 2.6$ years; height: $165.8 \pm 6.2 \mathrm{~cm}$; body mass: $61.4 \pm$ $8.9 \mathrm{~kg}$ ] volunteered to participate in this study. Test procedures were outlined in a written information sheet and were explained to participants before obtaining written informed consent. Experimental procedures were approved by Loughborough University Ethical Committee.

\subsection{Experimental design}

To achieve the aims of the study a repeated measures design was selected. Participants took part in two running trials on separate occasions, wearing running shoes of the same model but different in terms of air permeability (OPEN upper construction vs CLOSED upper construction). No information was provided to participants regarding shoe related differences and participants were not allowed to visually inspect the shoes. It is important to note however that some differences may have been noted when donning the shoes.

\subsection{Instrumentation and Calculations}

\subsubsection{Gross sweat loss and sock sweat absorption}

To determine gross sweat loss (GSL), pre- and post-test semi-nude body mass and fluid consumed during each experimental session were recorded using electronic scales with a resolution of $0.001 \mathrm{~kg}$ (Mettler Toledo kcc150, Mettler Toledo, Leicester, UK). GSL is presented in kilograms ( $\mathrm{kg})$ and was calculated:

$$
G S L(k g)=w_{b 1}-w_{b 2}+\text { fluid }
$$

Where;

$w_{b 1}$ body weight at start of experiment $(\mathrm{kg})$

$w_{b 2}$ body weight at end of experiment $(\mathrm{kg})$

total fluid consumption $(\mathrm{kg})$

Sock mass was recorded pre- and post-test using electronic scales with a resolution of $0.001 \mathrm{~g}$ (FX-500i. A\&D Company Ltd, Oxfordshire, UK) to determine sweat absorption into the sock. 


\subsubsection{Aural, mean skin temperature and heart rate}

Aural temperature $\left(T_{a u}\right)$ was measured from the auditory canal (Braun ThermoScan 7 , IRT6520, Braun GmbH, Kronberg, Germany) at the beginning/end of each stage of the experimental protocol. Changes to core temperature were expected to be minimal and not affected by footwear. An infrared aural thermography sensor was therefore chosen to reduce unnecessary discomfort to participants.

Skin temperature was measured at four sites: calf, thigh, chest and upper arm using iButton wireless temperature loggers (Maxim, San Jose, USA) attached to the skin by $3 \mathrm{M}^{\mathrm{TM}}$ Transpore ${ }^{\mathrm{TM}}$ surgical tape (3M United Kingdom PLC). The weighted skin temperature $\left(\bar{T}_{S k}\right)$ of four sites was calculated using the below equation (Ramanathan 1964):

$\bar{T}_{s k}=\left(0.3 * T_{s k}\right.$ Upper arm $)+\left(0.3 * T_{s k}\right.$ Chest $)+\left(0.2 * T_{s k}\right.$ Thigh $)+\left(0.2 * T_{s k}\right.$ Calf $)$

Heart rate (HR) was measured during all tests using telemetry (Polar RS400sd,Polar Electro Oy,Kempele, Finland).

\subsubsection{Foot skin temperature}

Foot $T_{s k}$ was measured at seven sites on the right foot (Figure 1) using $\mathrm{T}$ type thermocouples (plantar thermocouples, 1/0.508 $\mathrm{mm}$ wire, 827-5978; dorsal thermocouples, 1/0.315 mm wire, 110-4469, RS Components Ltd, Corby, UK). Thermocouples were connected to a data logger (Grant Squirrel SQ2020, Grant Instruments Ltd, Cambridge, UK) which logged foot $T_{s k}$ every $10 \mathrm{~s}$. Thermocouples were calibrated prior to testing by placing the measuring junction of each thermocouple in a circulating water bath where temperature was monitored with a calibrated thermometer (resolution $0.1^{\circ} \mathrm{C}$ ). 


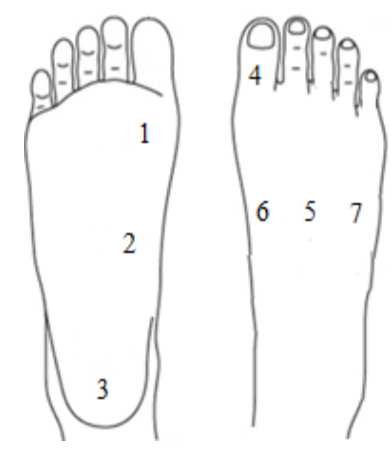

Figure 1 Anatomical positioning of thermocouples on the right foot. Site codes: 1-plantar ball; 2-plantar arch; 3- plantar heel; 4-base of toe 1; 5- central dorsal; 6-medial dorsal; 7lateral dorsal.

\subsubsection{In-shoe temperature and in-shoe relative humidity}

Temperature and relative humidity sensors were attached to seven sites on the right foot (Figure 2) to record changes to in-shoe temperature and in-shoe relative humidity (SHT31, Sensirion, Switzerland). Sensors were applied to a standardised sock using transpore surgical tape. Data was collected wirelessly from a specially developed bluetooth data acquisition system (University of Applied Sciences Kaiserslautern, Zweibrücken, Germany), secured to the participants ankle, at a sampling rate of $10 \mathrm{~s}$ and simultaneously displayed and recorded in LabVIEW software (2016, National Instruments). The total weight of the data acquisition system and sensors was $112 \mathrm{~g}$.
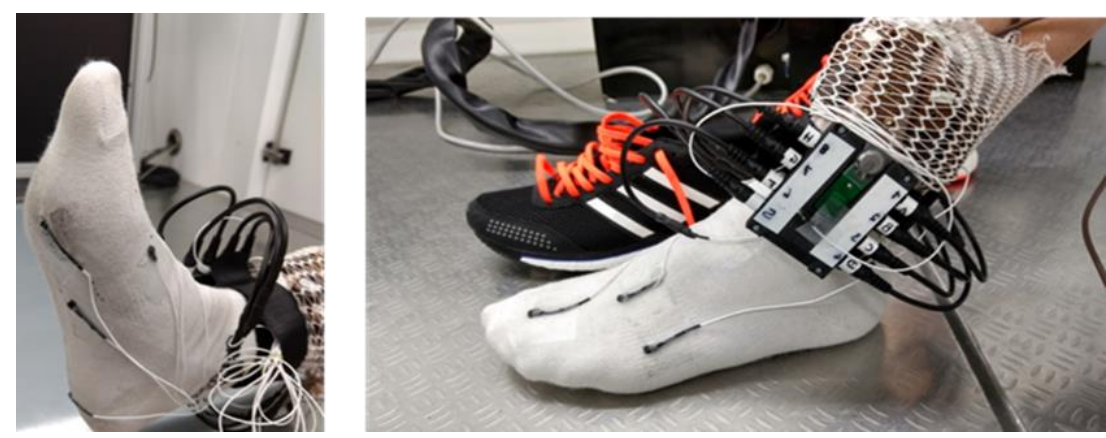

Figure 2 Positioning of temperature and relative humidity sensors on the right foot. 


\subsubsection{In-shoe absolute humidity}

In-shoe absolute humidity was calculated from in-shoe temperature and in-shoe relative humidity using the Antoine equation (Parsons 2014):

$$
\text { Absolute Humidity }\left(\text { g. } \mathrm{m}^{3}\right)=\frac{r h}{100} \times \frac{e^{\left[16.6536-\frac{4030.183}{T+235}\right]}}{461.4(T+273)} 10^{6}
$$

Where;

e exponential function

T temperature $\left({ }^{\circ} \mathrm{C}\right)$

rh relative humidity (\%)

\subsubsection{Perceptual scales}

Ordinal scales were used to assess thermal sensation, wetness perception, stickiness and thermal comfort (Figure 3). Scales were designed in line with instructions from ISO 10551 (2001) with the sensitivity, accuracy, ease of use and descriptors chosen for each individual scale carefully considered.

Scales were used to gain subjective information for the whole body (thermal sensation and thermal comfort), whole foot and for four foot regions (thermal sensation, wetness perception, stickiness and thermal comfort; Figure 4). Subjective information was obtained for the right foot only.

Participants were instructed on how to use the perceptual scales and were given time to practice under the guidance and assistance of the experimenter. During the experimental trial, participants were prompted every 5 mins to provide perceptual ratings which took between 1-2 mins. 

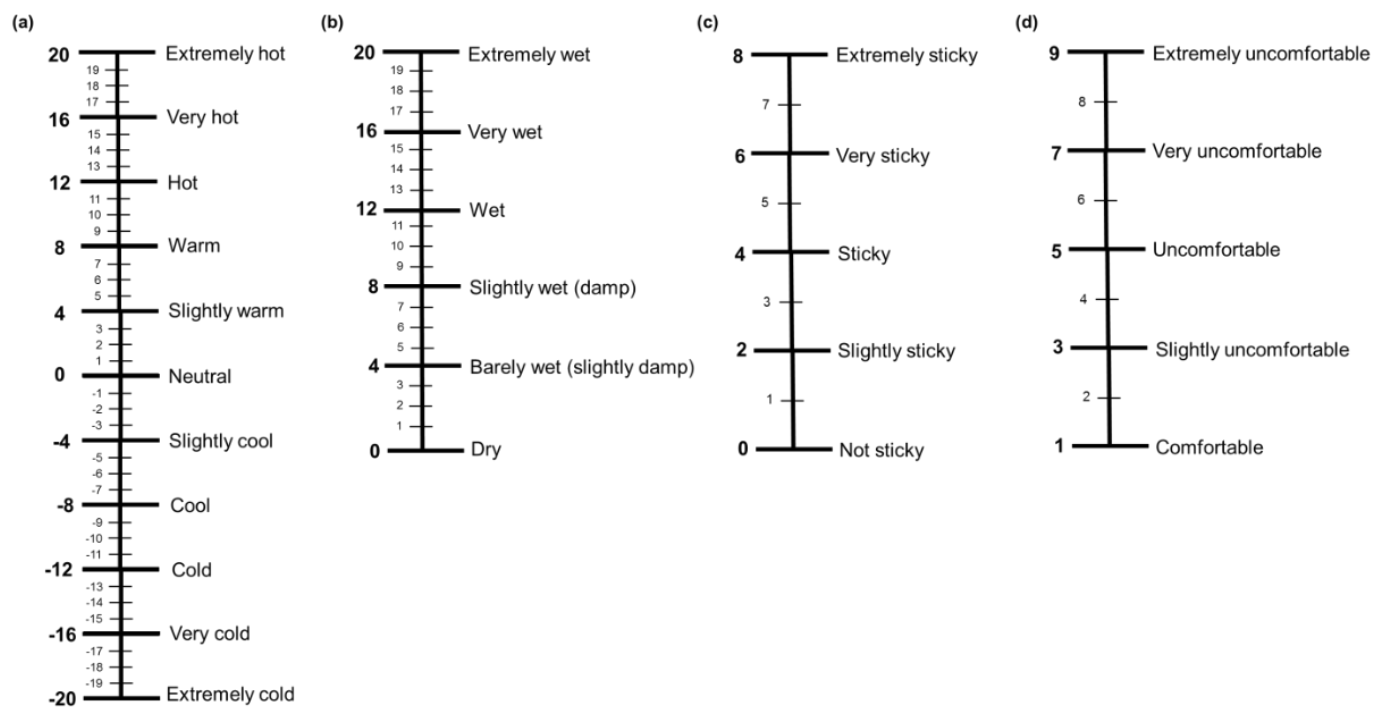

Figure 3 (a) thermal sensation scale; (b) wetness perception scale; (c) stickiness scale; (d) thermal comfort scale.

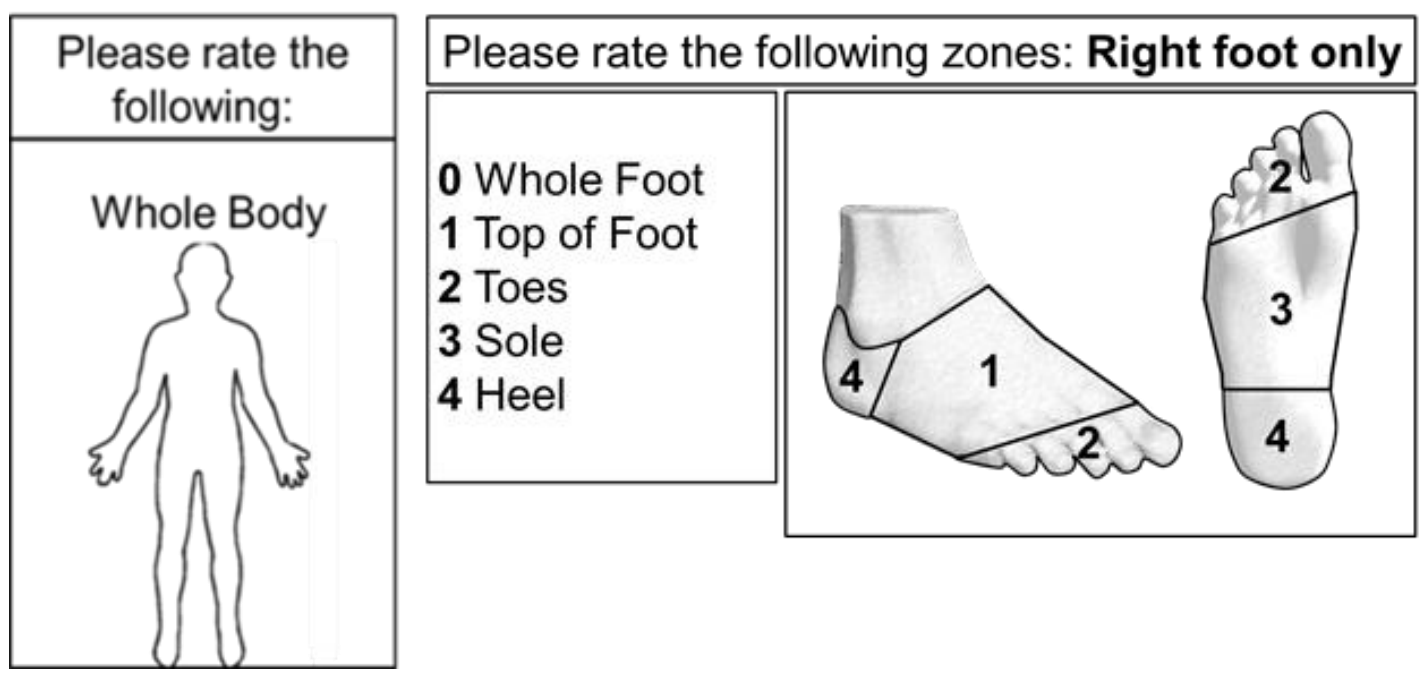

Figure 4 Reference sheet used to highlight areas for subjective evaluation.

\subsection{Experimental protocol}

Participants took part in two experimental trials at approximately the same time of day. Trials were performed in a climate controlled room maintained at $23^{\circ} \mathrm{C}, 35 \%$ relative humidity. The two experimental trials differed with regard to the running shoes worn (Figure 5). All participants performed one trial wearing a shoe with an OPEN air permeable upper construction (adidas Supernova Glide Boost 8 Clima Chill) and one trial wearing a shoe with a CLOSED less air permeable upper construction (adidas Supernova Glide Boost atr Clima Heat). Heat loss measurements for both shoes were performed using a 12 zone sweating thermal foot manikin (THERMETRICS, 
Measurement Technology Northwest, Seattle, USA) in accordance with ISO 15831 (2004). Thermal insulation was $0.10 \mathrm{~m}^{2} . \mathrm{K} \mathrm{W}^{-1}$ vs $0.11 \mathrm{~m}^{2} . \mathrm{K} . \mathrm{W}^{-1}$ and the evaporative resistance was $16.06 \mathrm{~m}^{2} . \mathrm{Pa} . \mathrm{W}^{-1}$ vs $25.18 \mathrm{~m}^{2} . \mathrm{Pa} . \mathrm{W}^{-1}$ for the OPEN and CLOSED shoe respectively. Socks were standardised for each trial (adidas performance ankle sock; $94 \%$ cotton, $3 \%$ polyamide, $3 \%$ elastane, terry jersey).

Upon arrival, participants changed into running shorts and t-shirt and washed their feet with tepid water. Following instrumentation, participants donned test shoes and rested in an upright seated position for a 10 min period. Participants then performed $40 \mathrm{~min}$ running at a constant speed $\left(7.5 \mathrm{~km} \cdot \mathrm{hr}^{-1}\right)$. This was followed by a $30 \mathrm{~min}$ recovery period in an upright seated position.

(a)

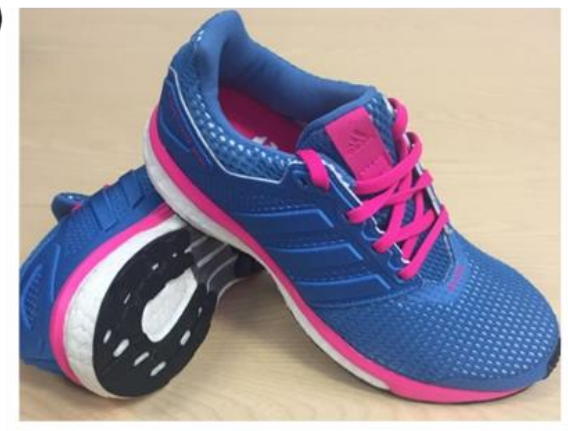

(b)

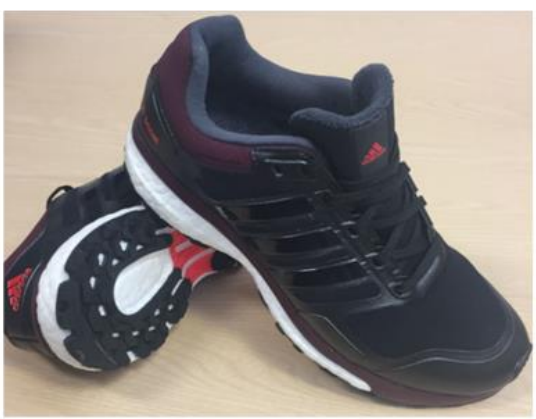

Figure 5 Running footwear worn during the experimental trials. (a) adidas Supernova Glide Boost 8 Clima Chill (OPEN) (b) adidas Supernova Glide Boost atr Clima Heat (CLOSED).

\subsection{Analysis}

The mean foot response for each individual variable (foot $T_{s k}$, in-shoe temperature and in-shoe relative humidity/absolute humidity) was calculated by averaging the data recorded from seven foot measurement sites for each participant over time and taken forward for statistical analysis.

To determine changes to foot $T_{s k}$, in-shoe temperature and in-shoe relative humidity/absolute humidity for different foot regions (dorsal, toes, heel and sole) data recorded from measurement sites which were representative of each region were averaged to calculate a mean response for each region. With reference to Figure 1 and Figure 2, measurement sites 5,6 and 7 were grouped to determine mean variable responses for the dorsal region. Measurement sites 1 and 2 were grouped for the sole 
region. Data from site 4 was taken to represent the toe region and data from site 3 was taken to represent the heel region.

\subsection{Statistical analysis}

In this study the independent variables were: shoe (OPEN vs CLOSED) and time (REST, RUN, RECOVERY). Dependent variables were: GSL, sock sweat absorption, HR, $T_{a u}, \bar{T}_{s k}$, foot $T_{s k}$, in-shoe temperature, in-shoe relative humidity, in-shoe absolute humidity, whole body thermal sensation and whole body thermal comfort and thermal sensation $(T S)$, wetness perception $(W P)$, stickiness $(S T)$ and thermal comfort $(T C)$ for the whole foot and by foot regions. Data were tested for normality of distribution with Shapiro-Wilk test.

To investigate temporal and spatial characteristics of shoe microclimate and to determine whether shoe microclimate is affected by shoe permeability a two way repeated measure analysis of variance (ANOVA) was performed with post hoc multiple comparisons (Bonferroni correction).

To investigate subjective perception of shoe microclimate over time and between shoe conditions a Friedman test was conducted. When significant effects were observed, post hoc analysis was conducted with a Wilcoxon signed rank test.

Regression analyses were performed to study the relationships between dependent and independent variables. These analyses were conducted using data from group means over time.

Statistical analysis was performed using IBM SPSS Statistics 24 (IBM, USA) and reported as means \pm standard deviation (SD). The level of significance was set at $p<$ 0.05 . 


\section{RESULTS}

\subsection{Environmental conditions}

Mean $( \pm \mathrm{SD})$ environmental conditions for the experimental trials were $23.2 \pm 0.2^{\circ} \mathrm{C}$ and $34.6 \pm 1.6 \% \mathrm{RH}$.

\subsection{Whole body thermal responses}

\subsubsection{Aural temperature, mean skin temperature and heart rate}

Whole body thermal responses highlighted no shoe related differences in terms of $\bar{T}_{s k}$, $T_{a u}$ or HR. $\bar{T}_{s k}$ was similar between shoe conditions throughout the experimental protocol (OPEN vs CLOSED: $31.4 \pm 0.5^{\circ} \mathrm{C}$ vs $31.3 \pm 0.5^{\circ} \mathrm{C}$ at REST; $30.9 \pm 0.9^{\circ} \mathrm{C}$ vs $31.1 \pm 2.1^{\circ} \mathrm{C}$ at the end of RUN; $31.5 \pm 0.9^{\circ} \mathrm{C}$ vs $31.8 \pm 1.2^{\circ} \mathrm{C}$ at the end of RECOVERY, all NS). Dynamics of $\bar{T}_{s k}$ were similar between shoe conditions.

There were no shoe-related differences in the dynamics of $T_{a u}$ and their absolute values (OPEN vs CLOSED: $37.1 \pm 0.3^{\circ} \mathrm{C}$ vs $37.1 \pm 0.4^{\circ} \mathrm{C}$ at REST; $36.9 \pm 0.8^{\circ} \mathrm{C}$ vs $37.0 \pm 0.8^{\circ} \mathrm{C}$ at the end of RUN; $37.1 \pm 0.6^{\circ} \mathrm{C}$ vs $37.1 \pm 0.5^{\circ} \mathrm{C}$ at the end of RECOVERY, all NS). Moreover, there were no differences in HR with a similar plateau during RUN at $143 \pm 19 \mathrm{bpm}$ for OPEN and $144 \pm 20 \mathrm{bpm}$ for CLOSED.

\subsubsection{Gross sweat loss and sock sweat absorption}

GSL was not different between OPEN $(0.46 \pm 0.10 \mathrm{~kg})$ and CLOSED $(0.46 \pm 0.19 \mathrm{~kg})$ shoe conditions $(p=0.95)$. No difference in sock sweat absorption was observed (OPEN vs CLOSED $1.60 \mathrm{~g}$ [min-max 0.60-7.10 g] vs $1.79 \mathrm{~g}$ [min-max 0.70-8.00 g], $p=0.66)$.

\subsubsection{Perceptual responses}

The dynamics of perceptual responses were similar between shoe conditions (NS). During RUN, whole body thermal sensations increased from neutral to warm, for OPEN (from $1.1 \pm 1.8$ at the end of REST to $11.3 \pm 4.6$ at the end of RUN, $p=0.01$ ) and CLOSED (from $1.5 \pm 2.8$ at the end of REST to $12.3 \pm 3.5$ at the end of RUN, $p$ $=0.01$ ). Consequently, participants developed moderate thermal discomfort during the running period.

With RECOVERY, whole body thermal sensations returned toward neutral for OPEN and CLOSED (from 11.3 \pm 4.6 and 12.3 \pm 3.5 at the end of RUN to $3.3 \pm 2.2$ and 4.6 \pm at the end of RECOVERY, $p<0.01$ ) resulting in reduced thermal discomfort (OPEN 
and CLOSED: from end of RUN $3.5 \pm 1.8$ and $3.9 \pm 2.1$ to end of RECOVERY $1.2 \pm$ 0.4 and $1.4 \pm 0.7, p<0.05)$.

Despite no shoe related differences in terms of $\bar{T}_{s k}$ or $T_{a u}$, participants felt warmer during the CLOSED shoe condition reaching significance at $45 \mathrm{mins}(p=0.02)$. There were no shoe related differences in whole body thermal comfort.

\subsection{Foot thermal responses}

\subsubsection{Mean foot skin temperature and mean in-shoe temperature}

Mean foot $T_{s k}$ (Figure 6a) increased with REST for both shoe conditions $(p<0.001)$. At the end of RUN (50 mins), mean foot $T_{s k}$ was $0.5^{\circ} \mathrm{C}$ greater for CLOSED (OPEN vs CLOSED: $36.6 \pm 0.7^{\circ} \mathrm{C}$ vs $37.1 \pm 0.7^{\circ} \mathrm{C}, \mathrm{p}=0.002$ ). With RECOVERY, mean foot $T_{s k}$ decreased by approximately $2^{\circ} \mathrm{C}$ regardless of SHOE condition $(p<0.001)$. Overall the difference in foot $T_{s k}$ between the two shoes reduced and lost significance over time.

In-shoe temperature (Figure 6b) increased with REST for both shoe conditions $(p<$ 0.001). At the end of RUN, in-shoe temperature was $0.9^{\circ} \mathrm{C}$ greater for CLOSED (OPEN vs CLOSED: $35.2 \pm 0.6^{\circ} \mathrm{C}$ vs $36.1 \pm 0.5^{\circ} \mathrm{C}, \mathrm{p}<0.001$ ). At the cessation of running (50-55 mins) in-shoe temperature briefly increased in the OPEN shoe but only by $0.3^{\circ} \mathrm{C}$.

\subsubsection{Mean in-shoe relative humidity and mean in-shoe absolute humidity}

Mean in-shoe relative humidity (Figure 6c) increased with REST for both shoe conditions ( $p<0.001$ ). At the end of RUN, in-shoe relative humidity was $8.2 \%$ greater for CLOSED (OPEN vs CLOSED: $75.8 \pm 7.9 \%$ vs $84.1 \pm 5.8 \%, \mathrm{p}<0.001$ ). With the cessation of running (50-55 mins) mean in-shoe relative humidity increased by $7.8 \%$ for OPEN and 3.8\% for CLOSED. Mean in-shoe relative humidity remained high during RECOVERY reaching $84.3 \pm 8.0 \%$ for OPEN and $87.5 \pm 6.7 \%$ for CLOSED at $80 \operatorname{mins}(p<0.05)$.

Mean in-shoe absolute humidity (Figure 6d) increased with REST and throughout RUN reaching $31.3 \pm 3.3$ g. $\mathrm{m}^{-3}$ for OPEN and $35.7 \pm 2.6 \mathrm{~g} \cdot \mathrm{m}^{-3}$ for CLOSED at $50 \mathrm{mins}$ ( $p$ < 0.001). With the cessation of running (50-55 mins), in-shoe absolute humidity increased briefly by 3.1 g.m $\mathrm{m}^{-3}$ for OPEN and $0.9 \mathrm{~g} . \mathrm{m}^{-3}$ for CLOSED. During RECOVERY (50-80 mins), in-shoe absolute humidity decreased by $2.4 \mathrm{~g} \cdot \mathrm{m}^{-3}$ for 
CLOSED only $(p<0.001)$. Given the lower temperature and the lower relative humidity in OPEN, the difference in absolute humidity is even stronger than that of relative humidity $(p<0.05)$. 


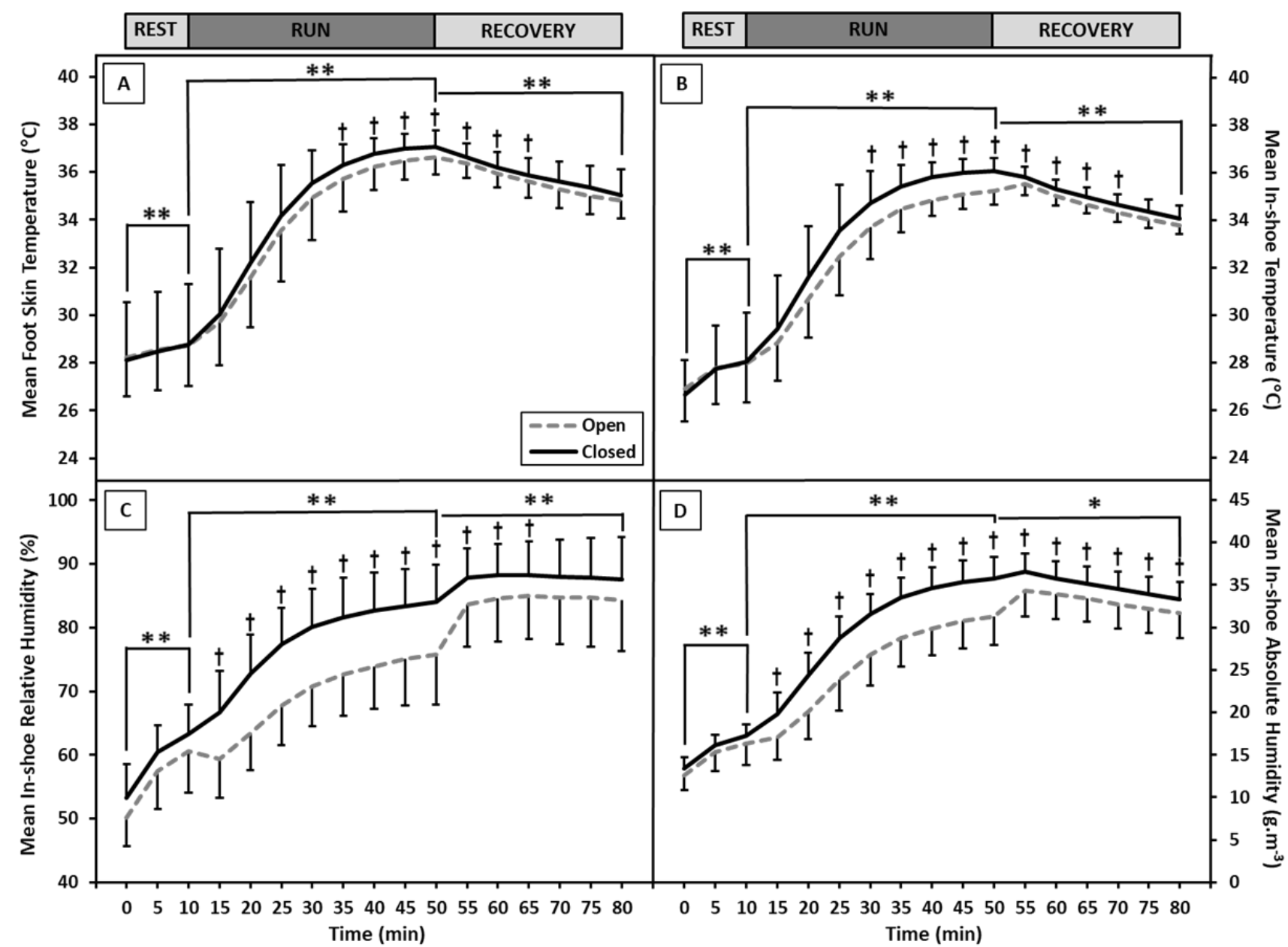

Figure 6 Mean (a) foot skin temperature, (b) in-shoe temperature, (c) in-shoe relative humidity and (d) in-shoe absolute humidity for two shoe conditions during the experimental protocol consisting of rest, run and recovery. $* *$ significant difference from the previous protocol phase for both shoe conditions $(p<0.05)$. * significant difference from previous protocol phase for CLOSED $(p<0.001) \dagger$ significant difference from OPEN $(p<0.05)$. 


\subsection{Foot perceptual responses}

\subsubsection{Mean foot thermal sensation}

With REST, participants TS (Figure 7a) were neutral with no change over time. During RUN, participants TS increased from neutral to warm/hot $(p<0.01)$. During RECOVERY, TS decreased to slightly warm $(p<0.01)$. Mean foot TS in CLOSED were significantly warmer than in OPEN during most of the running phase $(p<0.05)$.

\subsubsection{Mean foot wetness and stickiness}

Participants experienced no WP (Figure 7b) or ST (Figure 7d) at REST. At the end of RUN, perceptions of $W P$ and ST reached a mean of slightly wet and sticky. During RECOVERY, WP and ST decreased towards a mean of barely wet and slightly sticky respectively, for both OPEN and CLOSED $(p<0.05)$. Significant differences in WP between OPEN and CLOSED during RUN were observed at time points $25 \mathrm{~min}$ ( $p=$ $0.02), 30 \mathrm{~min}(p=0.02)$ and $35 \mathrm{~min}(p=0.01)$. Significant differences in ST between OPEN and CLOSED were observed from 20-45 mins and at $80 \mathrm{~min}(p<0.05)$.

\subsubsection{Mean foot thermal comfort}

With REST, participants were comfortable (Figure 7c) with no change over time. Participants experienced moderate local discomfort throughout RUN regardless of SHOE $(p<0.01)$. With RECOVERY, TC returned towards comfortable $(p<0.01)$. The effect of SHOE on TC was significant at time points $30 \mathrm{~min}(p=0.04)$ and $35 \mathrm{~min}$ $(p=0.03)$. 


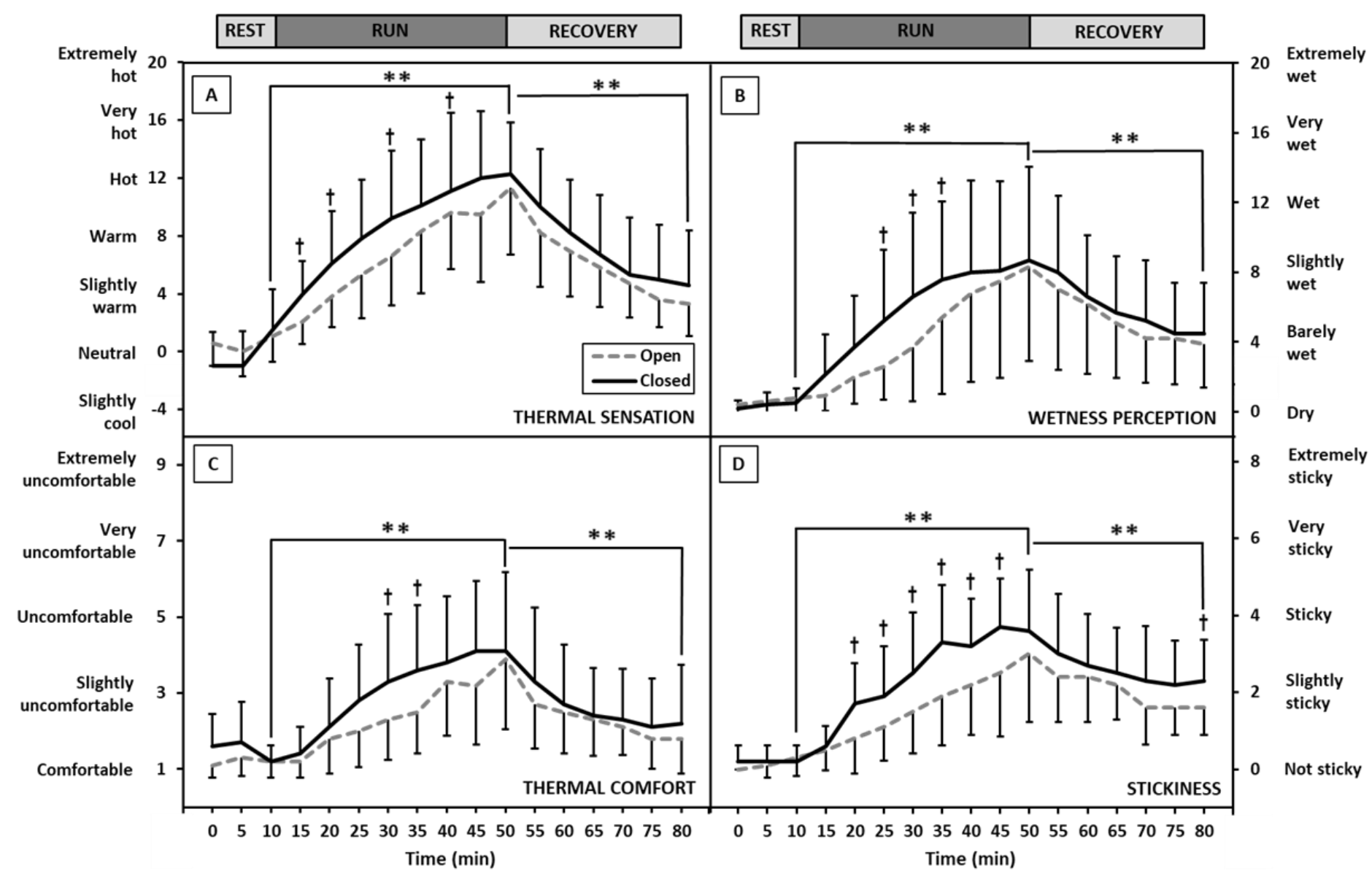

Figure 7 Mean (a) foot thermal sensation, (b) foot wetness perception, (c) foot stickiness and (d) foot thermal comfort for two shoe conditions across all stages of the experimental protocol consisting of rest, run and recovery. $* *$ significant difference from the previous protocol phase for both shoe conditions $(p<0.01)$. $\dagger$ significant from OPEN $(p<0.05)$. 


\subsection{Relation between foot thermal responses}

During RUN, positive relationships were observed between mean in-shoe relative humidity and mean in-shoe temperature (OPEN and CLOSED; $\mathrm{r}^{2}=0.97, p<0.001$ and $\left.r^{2}=1.00\right)$ and between mean in-shoe absolute humidity and mean in-shoe temperature (OPEN and CLOSED; $\mathrm{r}^{2}=0.99$ and $\mathrm{r}^{2}=1.00, p<0.001$ ).

With RECOVERY, a positive relationship was observed between in-shoe absolute humidity and mean in-shoe temperature (OPEN and CLOSED; $\mathrm{r}^{2}=0.98$ and $\mathrm{r}^{2}=1.00$, $p<0.001)$. The relationship between in-shoe relative humidity and in-shoe temperature however was weak and not significant (OPEN and CLOSED; $r^{2}=0.22$ and $\left.\mathrm{r}^{2}=0.28\right)$.

\subsection{Relation between foot perceptual responses and foot thermal responses}

During RUN, a positive relation was observed between mean foot $T S$ and mean foot $T_{s k}$ (OPEN and CLOSED; $\mathrm{r}^{2}=0.95$ and $\mathrm{r}^{2}=0.97, p<0.001$ ) and between mean foot $T S$ and mean in-shoe temperature (OPEN and CLOSED; $\mathrm{r}^{2}=0.94$ and $\mathrm{r}^{2}=0.96, p<$ 0.001). With RECOVERY, positive relationships were observed between mean foot $T S$ and mean foot $T_{s k}$ (OPEN and CLOSED; $\mathrm{r}^{2}=1.00$ and $\mathrm{r}^{2}=0.95, p<0.001$ ) and between mean foot TS and mean in-shoe temperature (OPEN and CLOSED; $\mathrm{r}^{2}=0.99$ and $\mathrm{r}^{2}=0.96, p<0.001$ ), although a hysteresis effect was evident after exercise ceased (Figure 8).
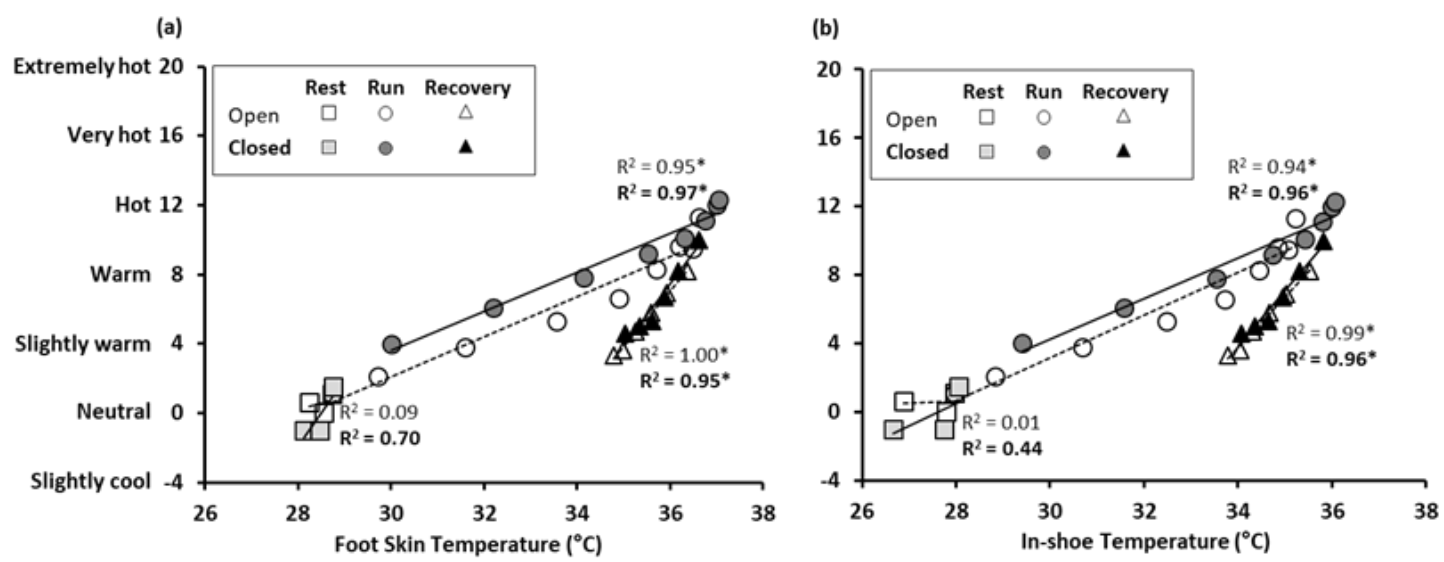

Figure 8 Relationship between mean foot thermal sensation and (a) mean foot skin temperature and (b) mean in-shoe temperature for two shoe conditions during an experimental protocol consisting of rest, run and recovery. *indicates a significant relationship $(p<0.001)$. 
During RUN, positive relationships were observed between mean foot $W P$ and mean in-shoe relative humidity and mean in-shoe absolute humidity. The relationship was tighter however with in-shoe absolute humidity (Figure 9).

During RECOVERY, no significant relation was observed between mean foot $W P$ and mean in-shoe relative humidity (OPEN and CLOSED; $\mathrm{r}^{2}=0.31, \mathrm{p}=0.247$ and $\mathrm{r}^{2}=$ $0.15, \mathrm{p}=0.450$; Figure 9). However positive relations were observed between mean foot $W P$ and mean in-shoe absolute humidity (OPEN and CLOSED; $\mathrm{r}^{2}=0.90, \mathrm{p}=$ 0.004 and $\left.r^{2}=0.94, p=0.001\right)$. A positive relation was also observed between mean foot $W P$ and mean foot $T_{s k}$ with less of a hysteresis evident (OPEN and CLOSED; $\mathrm{r}^{2}$ $=0.96$ and $\left.\mathrm{r}^{2}=0.95, \mathrm{p}<0.001\right)$. 
(a)

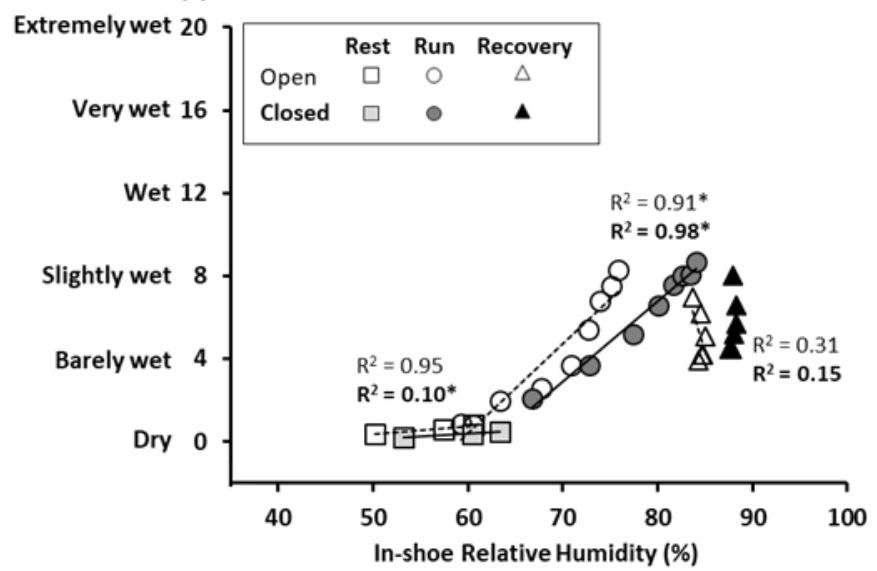

(b)

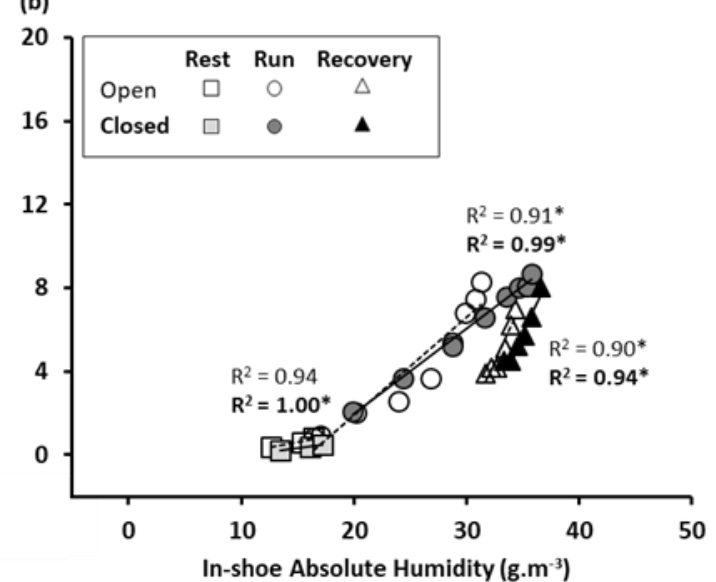

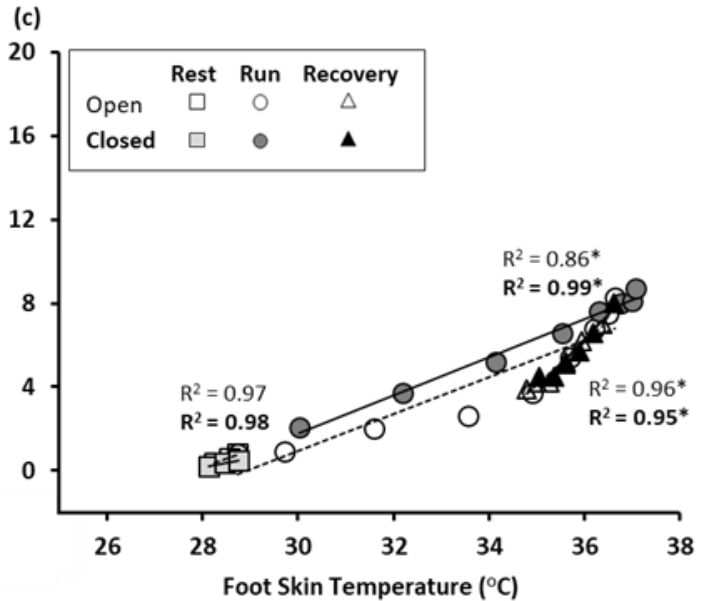

Figure 9 Relationship between mean foot wetness perception and (a) mean in-shoe relative humidity, (b) mean in-shoe absolute humidity and (c) mean foot skin temperature, for two shoe conditions during an experimental protocol consisting of rest, run and recovery. *indicates a significant relationship $(p<0.05)$. 
Mean foot $W P$ and mean foot $S T$ (Figure 10) were strongly linked during RUN and RECOVERY.

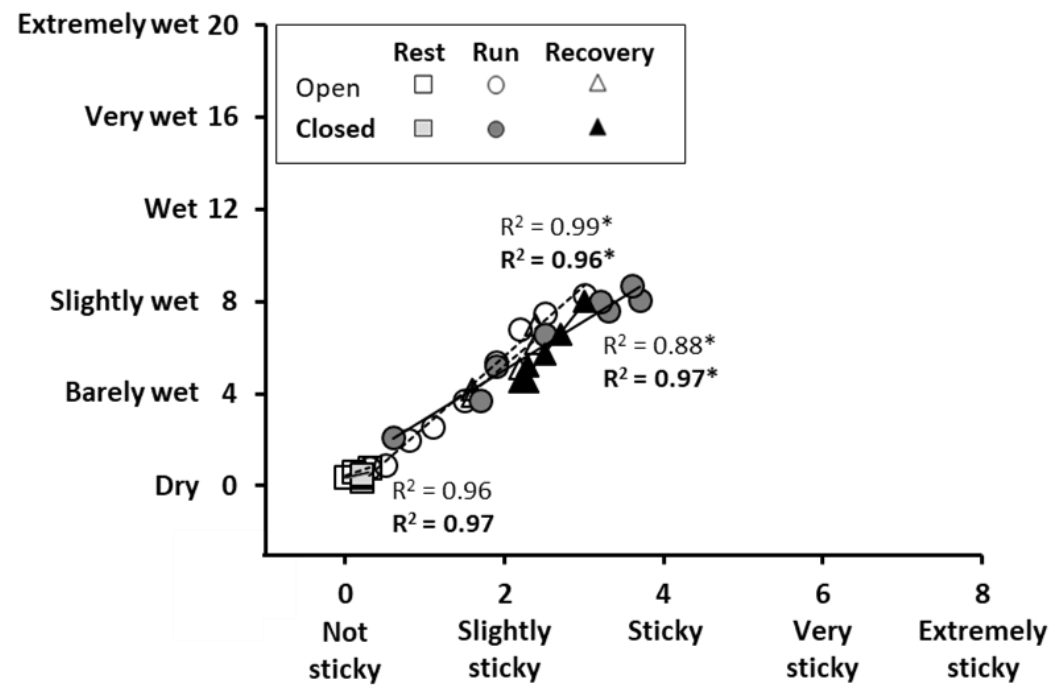

Figure 10 Relationship between mean foot wetness perception and mean foot stickiness for two shoe conditions during an experimental protocol consisting of rest, run and recovery. *indicates a significant relationship $(p<0.01)$.

To define factors affecting mean foot $T C$ when all stages of the experimental protocol (REST, RUN and RECOVERY) were combined, stepwise regression analysis was conducted. For this analysis, mean foot $T_{s k}$, mean in-shoe temperature, mean in-shoe relative humidity, mean in-shoe absolute humidity and $\bar{T}_{s k}$ were input as independent variables, and mean foot $T C$ as the dependent variable.

Mean foot $T C$ was described by mean foot $T_{s k}$ giving an explained variance of $67 \%$ (Table 1, Model 1). The inclusion of mean in-shoe relative humidity made a valuable improvement to the model $\left(\mathrm{r}^{2}=0.74, p<0.001\right)$ (Table 1, Model 2). Further improvements were observed with the additional inclusion of mean in-shoe absolute humidity and in-shoe temperature. However, tolerance statistics indicated collinearity $(<0.1)$ between independent variables, making that extended model questionable.

Given that several subjective scores, when merged over shoe types, had a tighter relation with in-shoe absolute humidity rather than relative humidity, the same analysis was performed forcing in-shoe absolute humidity as second predictor. This, combined with mean foot $T_{s k}$, explained less of the variance in mean foot $T C\left(\mathrm{r}^{2}=0.70, p<\right.$ 0.001), (Table 1, Model 3) than the relative humidity model, mainly due to the high co-linearity of the two variables used. 
Table 1 Stepwise regression analysis for mean foot thermal comfort when all phases of the experimental protocol (rest, run and recovery) are combined.

\begin{tabular}{|c|c|c|c|c|c|c|c|c|c|}
\hline \multirow[b]{2}{*}{ Perceptual Variables } & \multirow[b]{2}{*}{ Predictor Variable } & \multicolumn{2}{|c|}{$\begin{array}{c}\text { Unstandardised } \\
\text { coefficient }\end{array}$} & \multirow{2}{*}{ 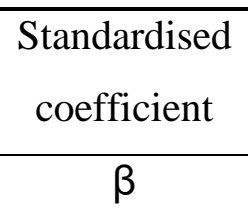 } & \multirow{2}{*}{$\begin{array}{c}\text { Collinearity } \\
\text { statistic }\end{array}$} & \multirow[b]{2}{*}{$\mathrm{t}$} & \multirow[b]{2}{*}{ Sign. } & \multirow[b]{2}{*}{$\mathrm{F}$} & \multirow[b]{2}{*}{$\mathrm{R}^{2}$} \\
\hline & & $\mathrm{B}$ & SD error & & & & & & \\
\hline MODEL 1 & (Constant) & -5.488 & 0.992 & & & -5.535 & $<0.001$ & 63.84 & 0.67 \\
\hline Thermal Comfort & Foot skin temperature & 0.233 & 0.029 & 0.816 & 1.000 & 7.990 & $<0.001$ & & \\
\hline MODEL 2 & (Constant) & -7.050 & 1.039 & & & -6.788 & $<0.001$ & 43.79 & 0.74 \\
\hline \multirow[t]{2}{*}{ Thermal Comfort } & Foot skin temperature & 0.378 & 0.056 & 1.326 & 0.218 & 6.744 & $<0.001$ & & \\
\hline & In-shoe relative humidity & -0.045 & 0.015 & -0.576 & 0.218 & -2.931 & $<0.001$ & & \\
\hline MODEL 3 & (Constant) & -9.910 & 2.485 & & & -3.987 & $<0.001$ & 36.48 & 0.70 \\
\hline \multirow[t]{2}{*}{ Thermal Comfort } & Foot skin temperature & 0.432 & 0.107 & 1.514 & 0.068 & 4.034 & $<0.001$ & & \\
\hline & In-shoe absolute humidity & -0.083 & 0.043 & -0.723 & 0.068 & -1.926 & 0.063 & & \\
\hline
\end{tabular}




\subsection{Local thermal responses by foot region}

Local foot thermal responses followed the general trend as shown for the mean foot skin temperature, mean in-shoe temperature, mean in-shoe relative humidity and mean in-shoe absolute humidity (Figure 6). Thus, only local foot in-shoe temperature and local foot in-shoe absolute humidity across foot regions are presented in Figure 11 for the OPEN and CLOSED shoe respectively.

\subsubsection{Mean local in-shoe temperature by foot region}

At the end of RUN and RECOVERY highest local in-shoe temperatures were observed at the heel and sole regions for both shoe conditions $(p<0.05)$ (Figure 11a and Figure 11b). In comparison to local foot $T_{s k}$, local in-shoe temperatures were lower at the dorsal and toe regions, but similar at the heel and sole regions reflecting low rates of heat transfer.

At the end of RUN, in-shoe temperature was significantly greater for CLOSED compared to OPEN at the dorsal, heel and toe regions $(p<0.05)$. At the end of RECOVERY, in-shoe temperature was significantly greater for CLOSED compared to OPEN at the toe region $(p=0.05)$.

\subsubsection{Mean local in-shoe absolute humidity by foot region}

At the end of RUN, highest local in-shoe absolute humidity's (Figure 11c and Figure 11d). were observed at the heel and sole regions for both shoe conditions $(p<0.05)$. In-shoe absolute humidity was significantly greater for CLOSED at the dorsal and toe regions $(p<0.01)$.

Local trends observed for changes in absolute humidity were similar to those of relative humidity across foot regions, excluding the recovery period. Local in-shoe relative humidity remained high (74-98\%) at all foot regions for both shoe conditions. However, local in-shoe absolute humidity significantly decreased for OPEN at the heel $(p=0.01)$ but significantly increased at the toes $(p=0.01)$ (Figure 11c). For CLOSED, local in-shoe absolute humidity significantly decreased at the sole $(\mathrm{p}=0.05)$, heel $(\mathrm{p}$ $=0.03)$ and toe $(\mathrm{p}<0.001)$ regions (Figure $11 \mathrm{~d})$. 


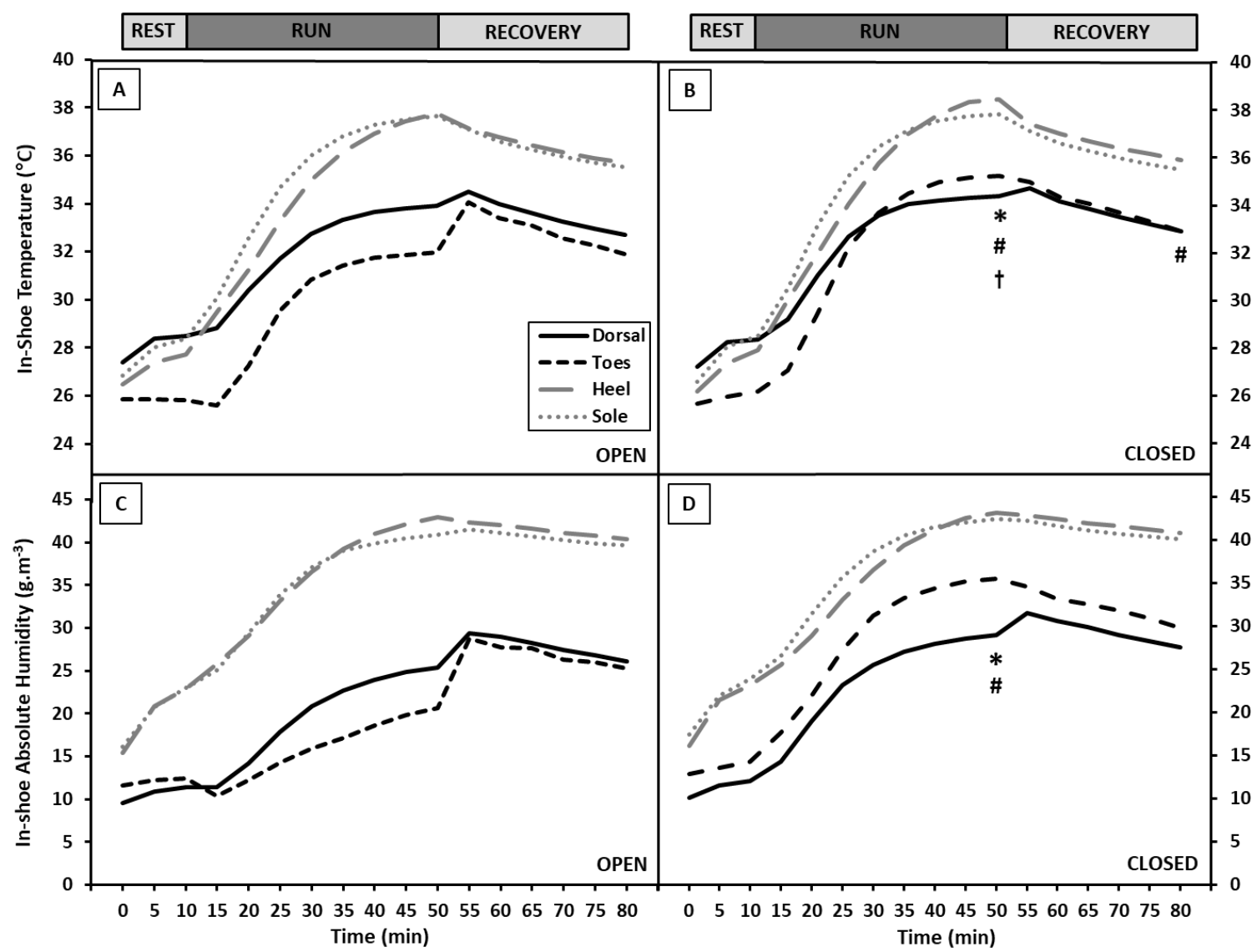

Figure 11 Mean (a) in-shoe temperature and (b) in-shoe absolute humidity across four foot regions (dorsal, toes, heel and sole) for two shoe conditions during the experimental protocol consisting of rest, run and recovery. * indicate significant difference from OPEN at the dorsal region. \# indicates significant difference from OPEN at the toe region. $\uparrow$ indicates significant difference from OPEN at the heel region. Significant differences $(p<$ 0.05) were also observed between foot regions for each shoe condition with the largest differences observed between the dorsal/toe and plantar regions. 


\subsection{Local perceptual responses by foot region}

\subsubsection{Mean local thermal sensation by foot region}

At the end of RUN, local TS (Figure 12a) at the toes (hot) was significantly greater than local TS at the dorsal (warm) $(p=0.03)$ for OPEN. No other significant differences in local TS between foot regions were observed. Local TS was significantly greater at the end of the RUN between OPEN and CLOSED at the dorsal $(\mathrm{p}=0.03)$ (Figure 12a and Figure 13a).

\subsubsection{Mean local wetness perception and mean local stickiness by foot region}

Local $W P$ and $S T$ (Figure 12b/d and Figure 13b/d) were significantly greater at the dorsal region at the end of REST compared to all other foot regions $(p<0.05)$. At the end of RUN, local $W P$ and ST was greater at the toes compared to the dorsal $(p<0.05)$ for OPEN. Local WP was greater at the toes compared to the heel $(p=0.04)$ region for both shoe conditions. Despite local in-shoe relative humidity increasing with the cessation of running and remaining close to saturation and in-shoe absolute humidity remaining close to/higher than local absolute humidity values experienced during the final 20 minutes of the running phase, perceptions of local WP and ST decreased with RECOVERY returning towards a mean of barely wet and slightly sticky at all regions $(p<0.05)$.

\subsubsection{Mean local thermal comfort by foot region}

With REST, participants rated all foot regions as being comfortable for both shoe conditions. Participants experienced moderate discomfort with RUN for all foot regions although higher discomfort was only experienced for the CLOSED shoe between the toe and dorsal region $(\mathrm{p}=0.03)$ (Figure 13c).

At the end of RUN, greater thermal discomfort was experienced for the CLOSED shoe compared to OPEN at the toe and heel region ( $\mathrm{p}<0.05)$. With RECOVERY, a significant decrease in thermal discomfort was observed at the dorsal, toes and sole for both shoe conditions $(p<0.05)$ and at the heel for CLOSED $(\mathrm{p}=0.01)$. 


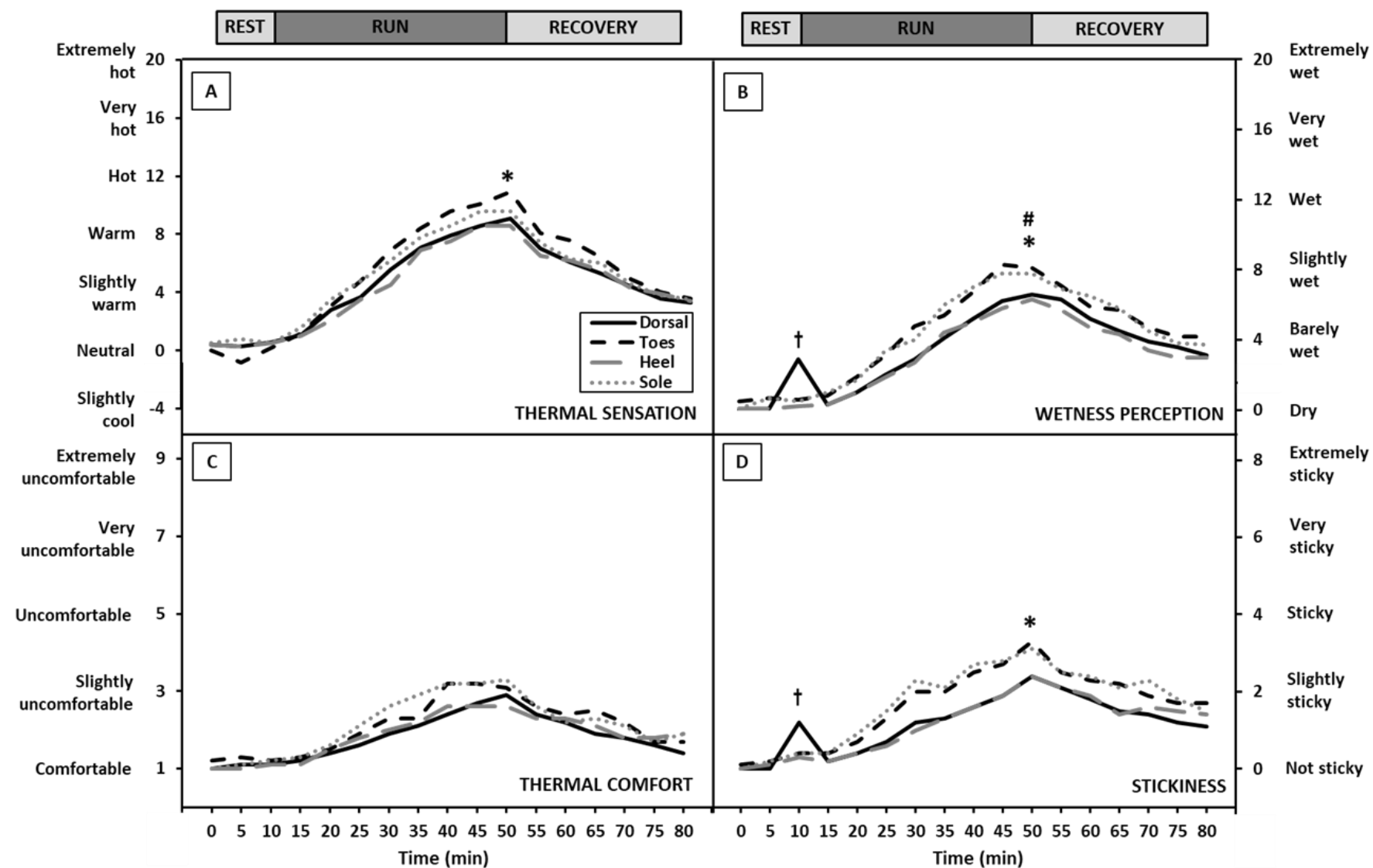

Figure 12 Mean (a) thermal sensation (b) wetness perception (c) thermal comfort and (d) stickiness across four foot regions (dorsal, toes, heel and sole) for the OPEN shoe condition during the experimental protocol consisting of rest, run and recovery.* indicates significant difference between the toe and dorsal region. \# indicates significant difference between the toe and heel region. $\dagger$ indicates significant difference between the dorsal and all remaining foot regions. 


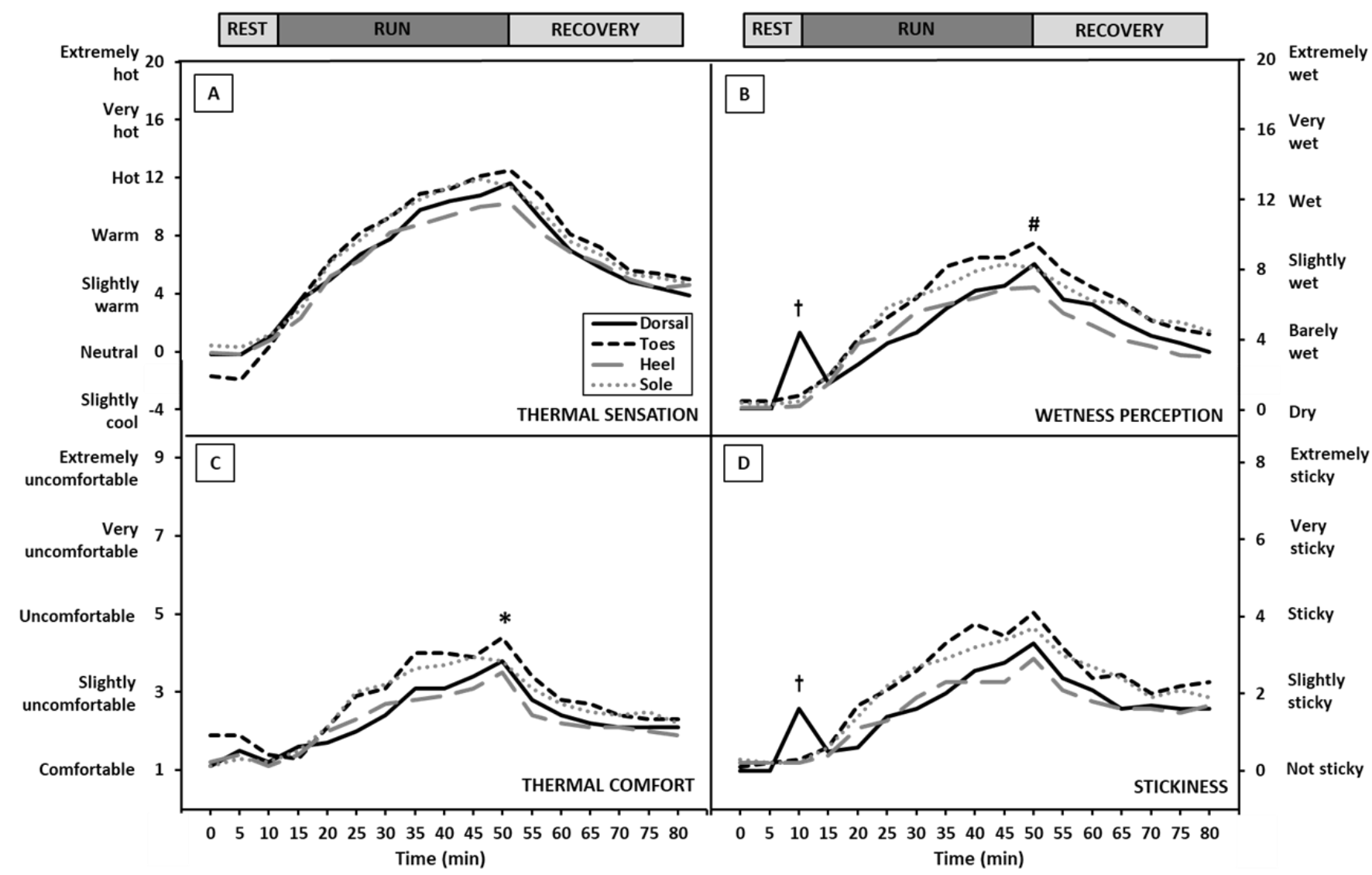

Figure 13 Mean (a) thermal sensation (b) wetness perception (c) thermal comfort and (d) stickiness across four foot regions (dorsal, toes, heel and sole) for the CLOSED shoe condition during the experimental protocol consisting of rest, run and recovery.* indicates significant difference between the toe and dorsal region. \# indicates significant difference between the toe and heel region. $\dagger$ indicates significant difference between the dorsal and all remaining foot regions. 


\section{Discussion}

The aim of this study was to objectively investigate the temporal and spatial characteristics of shoe microclimate in running footwear, to determine whether shoe microclimate is affected by shoe permeability and to determine whether changes to shoe microclimate are perceivable.

The outcomes of this study demonstrate that the general temporal characteristics of shoe microclimate variables were similar for both shoes assessed. However, a clear impact of the shoe permeability on shoe microclimate was observed particularly during running. The closed shoe (less air and vapour permeable) resulted in higher temperatures and levels of moisture accumulation in comparison to the open shoe. During recovery, shoe microclimate readings of the two shoes converged towards lower temperature and humidity. This suggests that with the cessation of exercise, reduced foot movement causes a reduction in the pumping effect and shoe ventilation (Satsumoto et al. 2016). Consequently, heat loss from the shoe is reduced. As the effect of ventilation was greater for the open shoe, causing a difference between open and closed shoe during run, the cessation of the pumping effect during recovery brings the heat production and heat loss balance for both shoes closer together. Regarding perceptual responses, clear differences between the shoes were observed during running, with more favourable responses for open. With recovery, the convergence of shoe microclimate variables also resulted in a convergence of perceptual responses.

\subsection{Temporal characteristics of foot $T_{s k}$ and shoe microclimate development}

During the initial $10 \mathrm{~min}$ of the experimental protocol, participants were required to rest. Temperature and moisture increased slightly but significantly during this period for both shoe conditions. With running, foot $T_{s k}$ and in-shoe temperature increased rapidly and substantially during the first 20 mins before gradually stabilising. The level of moisture within the shoe also increased during the first 20 mins before gradually stabilising for the remaining 20 mins of the run. Consequently, significant increases in temperature and moisture were reported from the start to the end of running period.

According to Shimazaki and Murata (2015) shoe microclimate variables increase based upon '(1) expelled heat generated inside the body and (2) heat transfer to/from the footwear surface'. With physical activity, metabolic heat generation increases. While little metabolic activity is present in the foot itself, the increased body heat load 
results in changes to vasomotor tone causing increases in foot blood flow as the body attempts to balance heat gain with heat loss. Consequently, the increased delivery of warm blood to the foot causes elevations in foot $T_{s k}$ reducing the body core to skin temperature gradient, impacting the temperature profile within footwear (Shimazaki and Murata 2015). Although heat loss can be substantially aided through the evaporation of sweat, containment of the foot within the shoe negatively impacts evaporative heat transfer. Therefore heat input into the foot tends to exceed heat loss from the foot causing a progressive increase in foot temperature, foot $T_{s k}$ and shoe microclimate variables.

During the recovery period of the experimental protocol, foot $T_{s k}$ decreased, likely due to reduced blood flow to the foot or a reduction in blood temperature. While heat losses would become less with the cessation of movement, heat loss from the shoe was nevertheless greater than the reduced heat input to the foot resulting in a reduction of in-shoe temperature. With regard to changes to in-shoe relative humidity and in-shoe absolute humidity values during the recovery phase of the experimental protocol, an inverse effect was observed. In-shoe relative humidity increased during recovery compared to no change/ a decrease in absolute humidity. The decrease in absolute humidity indicates that moisture losses from the shoe are now greater than the moisture generated by the foot. The fact that in-shoe relative humidity increases is therefore solely caused by the decreasing temperature lowering the saturated water vapour pressure. In-shoe relative humidity therefore increases despite the decreasing water content. To our knowledge, no study has presented shoe microclimate in relation to changes to absolute humidity.

\subsection{Spatial characteristics of foot $\boldsymbol{T}_{s k}$ and shoe microclimate}

The heel and sole foot regions were highlighted as being areas of high temperature and high moisture with running thus suggesting low rates of heat and moisture transfer from the foot at these regions. Irzmanska et al. (2013) similarly reported a higher inshoe temperature at the plantar region $\left(37^{\circ} \mathrm{C}\right)$ compared to the dorsal region $\left(36^{\circ} \mathrm{C}\right)$ following 50-60 minutes of walking in protective footwear. It has been suggested that repeated compression loads on these areas during walking and running produces heat from the conversion of mechanical energy (Shimazaki and Murata 2015). The absorption of contact forces on the plantar foot regions is therefore suggested to result in temperature elevations although there is no direct evidence for this. More 
importantly, although foot sweat rates are greatest from the dorsal surface compared to the plantar surface ( $\sim 70 \%$ and $\sim 30 \%$ respectively) (Taylor et al. 2006; Smith et al. 2013), highly insulative materials used within the mid-sole section of the shoe reduce dry and evaporative heat loss from the plantar foot surface to the environment leaving only heat loss by ventilation. Assuming a similar internal heat input to all foot regions, this insulative effect could explain why the heel and sole were areas of high temperature and moisture accumulation.

\subsection{Influence of shoe permeability on foot $T_{s k}$ and shoe microclimate}

In the current study, shoe microclimate was shown to be significantly affected by shoe permeability. This was most evident during the running and recovery stages of the experimental protocol as the open shoe consistently had lower temperature and moisture values in comparison with the closed shoe. These differences were also more pronounced at the toe region where differences in shoe construction were most apparent. In running shoes, the microclimate is therefore dependent upon the level of permeability provided as earlier described for protective and hiking footwear (Arezes et al. 2013; Irzmańska 2015).

Foot $T_{s k}$ is usually higher than ambient and footwear surface temperature resulting in heat transfer through convective and radiative pathways. It would be expected that during running, convective heat transfer would be high due to increased air flow around the shoe in addition to movement of the foot within the shoe causing a pumping action forcing air into the environment (Havenith et al. 1990; Satsumoto et al. 2016). The mesh upper construction of the open shoe allowed effective convective heat transfer during running resulting in lower foot $T_{s k}$ and in-shoe temperature elevations. Indeed, a relationship has been reported between temperature elevations and ventilation suggesting lower temperature elevations with higher ventilation rates (Shimazaki et al. 2015). The effect of convective heat transfer is also visible when transitioning from running to recovery. With the cessation of running, air flow around the foot reduces. Consequently in-shoe temperature slightly increases initially in the open shoe indicating a reduction in convective heat transfer from the shoe to the environment.

With increased heat delivery to the foot through vasomotor adjustments, it could also be expected that local sweat rates would increase. Although there were no significant 
differences in sock sweat absorption between shoe conditions, in-shoe relative humidity and in-shoe absolute humidity values were greater for the closed shoe. It is suggested that increased ventilation can be effective in limiting an increase in skin moisture concentration (Ueda et al. 2006; Satsumoto et al. 2016). As a result, the mesh upper construction of the open shoe resulted in greater ventilation and therefore a reduction in the amount of moisture accumulated within the shoe.

\subsection{Perceptions of shoe microclimate}

Changes to shoe microclimate are suggested to cause strong sensations of discomfort. However, relatively few studies have assessed perceptual parameters in footwear (Barkley et al. 2011; Arezes et al. 2013; Irzmanska et al. 2013). In the current study, higher $T S, W P$, and $S T$ were reported for the closed shoe in comparison to the open shoe during running and recovery. This is in line with the observed shoe microclimate data being greater for the closed shoe. Consequently, the closed shoe was perceived as more uncomfortable during running. This has applied importance indicating a need for shoes which support heat dissipation and moisture removal during physical activity performed in neutral to warm conditions in order to improve perceptions of local TC.

It has long been acknowledged that during exposure to warm or hot environments, the level of skin wetness drives thermal discomfort (Gagge et al. 1967). However, it is unclear as to whether foot discomfort is attributed to elevations in temperature or to moisture accumulation within footwear (Arezes et al. 2013; Irzmanska et al. 2013).

Temperature elevations were well perceived in the current study as strong correlations between foot $T_{s k}$ and $T S$ and in-shoe temperature and $T S$ were observed during run and recovery. However, the observation of a hysteresis effect during recovery indicates that the perception of temperature at the foot, is not solely determined by changes to foot $T_{s k}$, in-shoe temperature or in-shoe humidity. The reduction in $\bar{T}_{s k}$ during recovery in addition to the decrease in foot $T_{s k}$ may therefore also influence foot $T S$.

With regard to moisture accumulation and perceptions of wetness, strong relationships were observed during run. However, during recovery perceptions of wetness reduced from a mean of slightly wet to a mean of barely wet for both shoe conditions, despite in-shoe relative and absolute humidity remaining higher/close to values experienced during the final 20 minutes of the running phase. Thus indicating a more complex interaction of relevant parameters. 
Skin cooling contributes significantly to the perception of wetness. Filingeri et al. (2013) measured drops in forearm skin temperature ranging between $1.4-4.1^{\circ} \mathrm{C}$ with a cooling rate of $0.14-0.41^{\circ} \mathrm{C} / \mathrm{s}$ with the application of cold-dry stimulus to the forearm evoking a clear wetness perception. In the current study however, foot $T_{s k}$ increased during the run, nevertheless resulting in greater perceptions of wetness for both shoe conditions. Participants were therefore able to sense skin wetness although they did not experience any cold or cooling sensations. This is contrary to previous reports that during static application of a warm-wet stimulus $\left(4^{\circ} \mathrm{C}\right.$ and $8^{\circ} \mathrm{C}$ above local skin temperature) no local wetness was perceived as no skin cooling and thus no cold sensations were experienced (Filingeri et al. 2015b). Furthermore, when skin cooling was observed during recovery, perceptions of wetness did not increase. It is however important to consider that foot $T_{s k}$ in the current study ranged between $35-37^{\circ} \mathrm{C}$. Therefore despite a $2{ }^{\circ} \mathrm{C}$ decrease in foot $T_{s k}$ during recovery, this probably would not have activated peripheral cold sensitive thermoreceptors which operate within the range of $20-30^{\circ} \mathrm{C}$ (Guyton 1991; Romanovsky 2006). Indeed, Filingeri et al. (2013) was able to elicit this response as the resting skin temperature of the forearm before the application of the cold-dry stimulus was $\sim 30^{\circ} \mathrm{C}$.

It is important to consider however that investigations so far have mainly focused on very local skin wetness perceptions evoked by static or dynamic applications of thermal and pre-wetted stimuli (Filingeri et al. 2013, 2015b; Raccuglia et al. 2017). Perceptions of wetness evoked during conditions of sweat-induced skin wetness rather than externally applied wetness may be dependent more on mechanosensory inputs such as stickiness rather than thermal inputs (Raccuglia et al. 2018), especially where the body part is free to move over the textile surface as in the case of the foot-sock interaction.

Bergmann Tiest et al. (2012) reported that dynamic as opposed to static interactions with wet materials, significantly decreased wetness discrimination thresholds. Dynamic exploration of wet materials therefore increases an individual's cutaneous sensitivity to wetness. Assessments under conditions of sweat-induced wetness while wearing clothing (tight vs loose fitting garment) also indicated that if no skin cooling occurs, restricting the repeated adhesion and movement of clothing on wet skin results in a reduction in the overall level of wetness perceived (Filingeri et al. 2015a). 
Due to the complex interactions between the foot, sock and shoe, mechanosensory stimuli could be particularly high. This is reflected in the current study as participants sensed an increase in stickiness during running with this being integrated as wetness. Thus, it could be expected that during recovery, perceptions of wetness decrease despite in-shoe relative/absolute humidity remaining high, due to a reduced magnitude of stimulation of cutaneous mechanoreceptors generated between the foot, sock and shoe. Although participants are often observed moving their feet around in their shoes when asked for wetness perceptions during rest/recovery, this may not be sufficient to increase cutaneous sensitivity to wetness. The results from this study highlight the importance of tactile cues/dynamic exploration in attempting to perceive wetness within footwear.

As to whether foot discomfort is attributed to elevations in temperature or to moisture accumulation within footwear, the findings of the present study suggest that when all phases of the experimental protocol are considered, $67 \%$ of the variance in local TC can be attributed to foot $T_{s k}$. However, as discussed, positive relationships were observed between shoe microclimate variables and also between shoe microclimate variables and perceptual responses. High collinearity between variables therefore suggests that it may be difficult to discriminate between temperature and moisture in natural wear tests, as opposed to externally applied thermal/pre-wetted stimuli. Changes to local TC must therefore be considered as a function of both temperature and moisture accumulation within the shoe. 


\section{Conclusion}

The outcomes of this study have both fundamental and applied importance. On the applied side, it has been demonstrated that the temporal characteristics of shoe microclimate development were similar for both shoes assessed. Clear differences in shoe microclimate were however evident during running due to the differences in shoe permeability, but these differences converge once exercise stops. Furthermore the heel and sole foot regions were identified as areas of high temperature and high moisture accumulation. On the fundamental side, results from this study demonstrate that changes to shoe microclimate over time and between shoe conditions are perceivable by the wearer.

The results from this study are important for footwear design as shoe designers and manufacturers can work towards developing shoes which better support heat dissipation and moisture removal. Shoes which better support heat dissipation and moisture removal may reduce the growth of microorganisms and thereby reduce the risk of odour development and poor foot health (i.e. tinea pedis and blister formation). This could be achieved by increasing moisture loss through changing shoe materials or increasing ventilation by making shoes more permeable. Ventilation openings in the sole and heel regions could be particularly advantageous.

\section{Acknowledgements}

The authors would like to acknowledge the continued support from the adidas FUTURE team during this study with special thanks to Dr Christian Heyde and Heiko Schlarb. The authors also thank those who participated in this experiment.

\section{Conflicts of interest}

The research presented was funded by the adidas FUTURE team, adidas AG, Germany and the Environmental Ergonomics Research Centre, Loughborough Design School, Loughborough University. The authors were fully responsible for the conduct of the trial and the data. 


\section{References}

Arezes PM, Neves MM, Teixeira SF, et al (2013) Testing thermal comfort of trekking boots: An objective and subjective evaluation. Appl Ergon 44:557565. doi: 10.1016/j.apergo.2012.11.007

Auger P, Marguis G, Joly J, Attye A (1993) Epidemiology of tinea pedis in marathon runners: prevalence of occult athlete's foot. Mycoses 36:35-41

Barkley RM, Bumgarner MR, Poss EM (2011) Physiological Versus Perceived Foot Temperature and Perceived Comfort during Treadmill Running in Shoes and Socks of Various Constructions. Am J Undergrad Res 10:7-14

Bergmann Tiest WM, Kosters ND, Kappers AML, et al (2012) Phase change materials and the perception of wetness. Ergonomics 55:508-512. doi: 10.1080/00140139.2011.645886

Bertaux E, Derler S, Rossi RM, et al (2010) Textile, Physiological, and Sensorial Parameters in Sock Comfort. Text Res J 80:1803-1810. doi: $10.1177 / 0040517510369409$

Bouskill LM, Havenith G, Kuklane K, et al (2002) Relationship between clothing ventilation and thermal insulation. AIHA J 63:262-268. doi: $10.1080 / 15428110208984712$

Filingeri D, Fournet D, Hodder S, Havenith G (2015a) Tactile cues significantly modulate the perception of sweat-induced skin wetness independently of the level of physical skin wetness. J Neurophysiol 113:3462-3473. doi: 10.1152/jn.00141.2015

Filingeri D, Redortier B, Hodder S, Havenith G (2015b) Warm temperature stimulus suppresses the perception of skin wetness during initial contact with a wet surface. Ski Res Technol 21:9-14. doi: 10.1111/srt.12148

Filingeri D, Redortier B, Hodder S, Havenith G (2013) The role of decreasing contact temperatures and skin cooling in the perception of skin wetness. Neurosci Lett 551:65-69. doi: 10.1016/j.neulet.2013.07.015

Gagge AP, Stolwijk JAJ, Hardy JD (1967) Comfort and thermal sensations and associated physiological responses at various ambient temperatures. Environ Res 1:1-20

Goonetilleke R, Luximon A (2001) Designing for comfort: a footwear application. In: Proceedings of the computer-aided ergonomics and safety conference 
Guyton AC (1991) Textbook of Medical Physiology

Havenith G (2002) Interaction of clothing and thermoregulation. Exog Dermatology 1:221-230. doi: 10.1159/000068802

Havenith G, Heus R, Lotens W A. (1990) Resultant clothing insulation: a function of body movement, posture, wind, clothing fit and ensemble thickness.

Ergonomics 33:67-84. doi: 10.1080/00140139008927094

Irzmańska E (2015) The Microclimate In Protective Fire Fighter Footwear: Foot

Temperature And Air Temperature And Relative Humidity. Autex Res J 1-5. doi: 10.1515/aut-2015-0030

Irzmanska E, Dutkiewicz JK, Irzmanski R (2013) New approach to assessing comfort of use of protective footwear with a textile liner and its impact on foot physiology. Text Res J 1-11. doi: 10.1177/0040517513507362

ISO15831 (2004) Clothing - Physiological effects - Measurement of thermal insulation by means of a thermal manikin

ISO 10551 (2001) Ergonomics of the thermal environment-assessment of the influence of the thermal environment using subjective judgement scales. International Standard, Geneva

Lu Y, Li J, Li X, Song G (2013) The effect of air gaps in moist protective clothing on protection from heat and flame. J Fire Sci 31:99-111. doi:

$10.1177 / 0734904112457342$

Parsons K (2014) Human thermal environments: the effects of hot, moderate, and cold environments on human health, comfort, and performance. Crc Press

Raccuglia M, Pistak K, Heyde C, et al (2017) Human wetness perception of fabrics under dynamic skin contact. Text Res J 4051751771690. doi: 10.1177/0040517517716905

Raccuglia M, Sales B, Heyde C, et al (2018) Clothing comfort during physical exercise - Determining the critical factors. Appl Ergon 73:33-41. doi: 10.1016/j.apergo.2018.05.014

Ramanathan N (1964) A new weighting system for mean surface temperature of the human body. J Appl Physiol 19:531-533

Romanovsky AA (2006) Thermoregulation: some concepts have changed . Functional architecture of the thermoregulatory system. Am J Physiol Regul Integr Comp Physiol 292:37-46. doi: 10.1152/ajpregu.00668.2006. 
Satsumoto Y, Piao S, Takeuchi M (2016) Effects of Shoe Fit and Moisture Permeability of a Leather Shoe on Shoe Microclimate and Air Exchange. J Ergon 6:1-7. doi: 10.4172/2165-7556.1000171

Schols EHM, van den Eijnde WAJ, Heus R (2004) A method for assessing thermal comfort of shoes using a “ sweating” foot. Eur J Appl Physiol 92:706-709. doi: 10.1001/jama.1930.02710480001001

Shimazaki Y, Matsutani T, Satsumoto Y (2015) Evaluation of thermal formation and air ventilation inside footwear during gait: The role of gait and fitting. Appl Ergon 1-7. doi: 10.1016/j.apergo.2015.11.002

Shimazaki Y, Murata M (2015) Effect of gait on formation of thermal environment inside footwear. Appl Ergon 49:55-62. doi: 10.1016/j.apergo.2015.01.007

Smith CJ, Machado-Moreira CA, Plant G, et al (2013) Design data for footwear: sweating distribution on the human foot. Int J Cloth Sci Technol 25:43-58. doi: $10.1108 / 09556221311292200$

Sulzberger MB, Cortese TA, Fishman L, Wiley HS (1966) Studies on blisters produced by friction. I. Results of linear rubbing and twisting technics. J Invest Dermatol 47:456-465. doi: 10.1038/jid.1966.169

Taylor NAS, Caldwell JN, Mekjavic IB (2006) The sweating foot: Local differences in sweat secretion during exercise-induced hyperthermia. Aviat Sp Environ Med $77: 1020-1027$

Ueda H, Inoue Y, Matsudaira M, et al (2006) Regional microclimate humidity of clothing during light work as a result of the interaction between local sweat production and ventilation. Int J Cloth Sci Technol 18:225-234. doi: $10.1108 / 09556220610668473$ 